\title{
WHAT'S NEW IN ICM
}

\section{Liberal versus restrictive fluid therapy in critically ill patients}

\author{
Jonathan A. Silversides ${ }^{1,2}$, Anders Perner ${ }^{3}$ and Manu L. N. G. Malbrain ${ }^{4,5^{*}}$ (1)
}

๑ 2019 Springer-Verlag GmbH Germany, part of Springer Nature

\begin{abstract}
Administration of intravenous fluid is one of the most commonly applied therapies in critical care, and has evolved from the replacement of water and electrolytes lost in diarrhoeal illness to the concept of 'optimising' cardiac output and thus, it is often incorrectly assumed as tissue perfusion [1]. As critical care transitions from a historical focus on normalisation of physiology towards the rigorous application of evidence-based practices which result in the best possible long-term outcomes, a re-evaluation of the role of basic therapies, such as intravenous fluids, is necessary.

Shock is typically described as hypovolaemic (e.g., haemorrhagic), distributive (e.g., septic), obstructive, cardiogenic, or neurogenic in origin, with frequent overlap. While careful consideration should be given to the aetiology of abnormal haemodynamics in the ICU with specific treatment directed at likely causes and, crucially, on the presence or absence of associated hypoperfusion, in our experience this is often not the case in practice. As hypovolaemia is the most readily reversible cause of shock, a 'liberal' approach using intravenous fluid boluses as the initial response to a variety of haemodynamic situations, including hypotension, tachycardia, oliguria, skin mottling, and elevated serum lactate levels is common $[2,3]$. This approach is consistent with international guidelines for the initial management of sepsis [4], and is often continued throughout the duration of critical illness, often without careful consideration of likely benefits versus harmful consequences [2].
\end{abstract}

*Correspondence: manu.malbrain@uzbrussel.be

${ }^{4}$ Department Intensive Care Medicine, University Hospital Brussels (UZB), Jette, Belgium

Full author information is available at the end of the article

\section{Efficacy of intravenous fluid administration}

While patients presenting to an emergency department (ED) may be profoundly hypovolaemic and initially respond to intravenous fluid, the effects diminish rapidly over time. In a recent randomised trial of lactate versus peripheral perfusion-guided fluid resuscitation in sepsis, less than $5 \%$ of patients were fluid responsive at $8 \mathrm{~h}$ from presentation (Fig. 1) [5]. This is also the case in patients admitted directly from the ED to ICU, by the time patients reach the ICU, therefore, fluid responsiveness is perhaps the exception rather than the rule. Indeed, the physiological effects of fluid boluses given in ICU appear to be small. In post hoc analyses of randomised trials in ARDS [6] and sepsis [7], the mean increase in blood pressure following a fluid bolus was in the order of $2 \mathrm{mmHg}$ and decrease in heart rate $1 \mathrm{bpm}$, with no change in urine output, and even these modest benefits often dissipate after as little as $1 \mathrm{~h} \mathrm{[8]}$. These data are in stark contrast to clinician-reported perceptions of efficacy in nearly $70 \%$ of fluid boluses [2].

\section{Potential harm from fluid administration}

In this context of large volume fluid intake, together with endocrine and renal responses which predispose to reduced water and salt excretion, the accumulation of a positive fluid and sodium balance is common. Fluid accumulation in critically ill patients is consistently associated with adverse outcomes. This has now been demonstrated in adults and children with sepsis, ARDS [9], and acute kidney injury (AKI) [10], as well as broader cohorts of critical illness [11]. The residual question is whether this represents a causal relationship, or merely confounding by indication, with more severely unwell patients being more likely to receive larger volumes of fluid and less likely to undergo measures to limit this fluid accumulation. A further question is whether the harm, if it exists, is due to the administration or to the accumulation of fluid

\section{Springer}




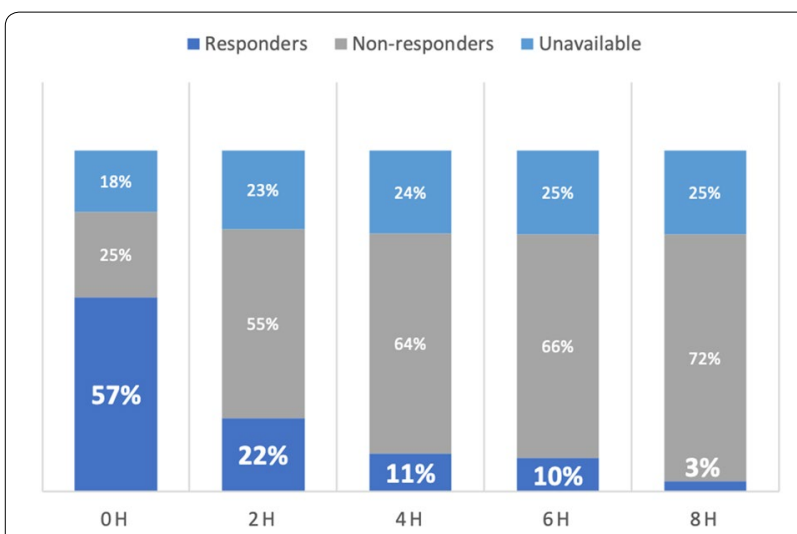

Fig. 1 Fluid responsiveness (in \%) over time in 424 patients with sepsis Adapted with permission from Hernandez et al. [5]

and sodium, since experimental data implicate both [12]. Potential mechanisms of harm from rapid fluid boluses include shearing injury to the endothelial glycocalyx, the fragile barrier by which fluid is maintained within the intravascular space, vasodilation, and decreased adrenergic responsiveness [13], while accumulation of fluid may result in haemodilution, elevated venous pressure with resultant decrease in perfusion pressure gradients, and interstitial oedema with resultant inhibition of oxygen diffusion between capillaries and cells [14].

Broadly speaking, two complementary approaches may be used in the prevention and treatment of fluid overload: restrictive fluid administration, and the active removal of accumulated fluid. The different phases following initial resuscitation and subsequent fluid removal are illustrated and explained in the ROSE model (ESM 1) [1].

\section{Restrictive fluid administration}

Considerable emphasis has been placed on identifying predictors of 'fluid responsiveness', i.e., physiological or echocardiographic parameters such as stroke volume variation [3] passive leg raise manoeuvres [15], or velocity time integral [16] to predict whether a fluid bolus will produce some improvement in haemodynamic variables. One rationale for these predictors is to limit fluid administration to situations where there is at least a possibility of clinical benefit.

While incorporating predictors of fluid responsiveness into overall haemodynamic assessment seems intuitively reasonable, inherent problems remain even if used correctly. First, administering fluid until a fluidunresponsive state is reached has never been shown to be of benefit in sepsis. Second, each of the predictors is subject to major limitations, for example, stroke volume variation is not useful in the context of lung-protective ventilation, spontaneous breathing, cardiac arrhythmias, right heart failure or increased intra-abdominal pressure [3]. Third, even if the prediction of fluid responsiveness is accurate, measures used to judge 'success' or 'failure' of a fluid bolus are global parameters such as blood pressure and cardiac output, which are poor predictors of tissue perfusion, particularly in sepsis where coherence between macro- and micro-circulation is frequently lost [14].

To reduce fluid administration, the early use of vasopressors may be needed. Early application of vasopressors has the potential to reduce intravascular volume deficit by recruiting blood from the venous compartment, while avoiding the detrimental effects of fluid administration [17]. Delaying vasopressor use until fluid resuscitation has demonstrably failed to correct shock, on the other hand, may delay resolution, and assumes that fluid resuscitation is safer than vasopressor use, an assumption which is contrary to the available data $[18,19]$ and which may be driven in part by resource constraints.

\section{Deresuscitation}

While more restrictive use of fluid, together with earlier use of vasopressors if needed, may reduce fluid administration, it is unlikely that fluid overload can be entirely avoided using this strategy. Fluid intake in ICU is from a range of sources, and many are obligatory such as drug diluents and nutrition. A recent study showed that this 'fluid creep' accounts for as much as 33\% of all fluid intake compared to $7 \%$ for resuscitation fluids [20]. Besides restriction of resuscitation fluids, avoidance of fluid overload is likely to require deresuscitation, defined as active fluid removal using diuretics or ultrafiltration [11], an approach which shortens the duration of mechanical ventilation and ICU stay [9]. Co-administration of hyperoncotic albumin along with diuretics may promote haemodynamic stability and diuresis [21, 22]. Ongoing work is focussed on three main areas: (1) restrictive approaches to fluid resuscitation, involving early use of vasopressors if needed, (2) the use of deresuscitative measures to prevent and treat fluid accumulation following resuscitation, and (3) the search for tools that may predict the patients who may benefit from fluid and those who do not. Meanwhile, clinicians should seek to avoid fluid administration where it is unlikely to be of benefit, i.e., where perfusion is adequate, even if vasopressors are needed, or when perfusion is inadequate but fluid responsiveness is unlikely. Following resuscitation, the use of diuretics or ultrafiltration to minimise fluid overload appears safe and may hasten recovery from critical illness. This is reflected in the dynamic phases (ROSE) of fluid management. Finally, while these represent broadly applicable principles, refinement of physiological measurements or biomarkers may allow a more 
personalised approach to fluid management in the future. In analogy to the well-known concept of antibiotic stewardship, it is thus time for fluid stewardship in the critically ill, where fluids should also be treated as drugs.

\section{Electronic supplementary material}

The online version of this article (https://doi.org/10.1007/s00134-019-05713-y) contains supplementary material, which is available to authorized users.

\section{Author details}

${ }^{1}$ Centre for Experimental Medicine, Queen's University of Belfast, Belfast, UK. 2 Department of Critical Care, Belfast Health and Social Care Trust, Belfast, UK. ${ }^{3}$ Department of Intensive Care, Rigshospitalet, University of Copenhagen, Copenhagen, Denmark. ${ }^{4}$ Department Intensive Care Medicine, University Hospital Brussels (UZB), Jette, Belgium. ${ }^{5}$ Faculty of Medicine and Pharmacy, Vrije Universiteit Brussel (VUB), Brussels, Belgium.

\section{Acknowledgements}

Dr. Manu Malbrain is a professor at the faculty of Medicine and Pharmacy at the Vrije Universiteit Brussels (VUB) and a member of the Executive Committee of the Abdominal Compartment Society, formerly known as the World Society of Abdominal Compartment Syndrome (https://www.wsacs.org/). He is the former president, co-founder and current Treasurer of WSACS. He is also the co-founder of the International Fluid Academy (IFA). The IFA is integrated within the not-for-profit charitable organization iMERiT, International Medical Education and Research Initiative, under Belgian law. The content of the IFA website (http://www.fluidacademy.org) is based on the philosophy of FOAM (Free Open Access Medical education-\#FOAMed). He is a member of the medical advisory Board of Pulsion Medical Systems (now fully integrated in Getinge, Solna, Sweden) and Serenno Medical (Tel Aviv, Israel), consults for Baxter, Maltron, ConvaTec, Acelity, Spiegelberg and Holtech Medical.

\section{Publisher's Note}

Springer Nature remains neutral with regard to jurisdictional claims in published maps and institutional affiliations.

Received: 23 June 2019 Accepted: 25 July 2019

Published online: 9 August 2019

\section{References}

1. Malbrain MLNG, Van Regenmortel N, Saugel B et al (2018) Principles of fluid management and stewardship in septic shock: it is time to consider the four D's and the four phases of fluid therapy. Ann Intensive Care 8:66

2. Cecconi M, Hofer C, Teboul J-L et al (2015) Fluid challenges in intensive care: the FENICE study: a global inception cohort study. Intensive Care Med 41:1529-1537

3. Van der Mullen J, Wise R, Vermeulen G et al (2018) Assessment of hypovolaemia in the critically ill. Anaesthesiol Intensive Ther 50:141-149

4. Rhodes A, Evans LE, Alhazzani W et al (2017) Surviving sepsis campaign: international guidelines for management of sepsis and septic shock: 2016. Intensive Care Med 43:304-377

5. Hernandez G, Ospina-Tascón GA, Damiani LP et al (2019) Effect of a resuscitation strategy targeting peripheral perfusion status vs serum lactate levels on 28-day mortality among patients with septic shock. JAMA 321:654-711

6. Lammi MR, Aiello B, Burg GT et al (2015) Response to fluid boluses in the fluid and catheter treatment trial. Chest 148:919-926

7. Bjerregaard MR, Hjortrup PB, Perner A (2019) Indications for fluid resuscitation in patients with septic shock: post-hoc analyses of the CLASSIC trial. Acta Anaesthesiol Scand 63:337-343

8. Glassford NJ, Eastwood GM, Bellomo R (2014) Physiological changes after fluid bolus therapy in sepsis: a systematic review of the contemporary literature. Crit Care 18:696

9. Silversides JA, Major E, Ferguson AJ et al (2017) Conservative fluid management or deresuscitation for patients with sepsis or acute respiratory distress syndrome following the resuscitation phase of critical illness: a systematic review and meta-analysis. Intensive Care Med 43:155-170

10. Prowle JR, Chua H-R, Bagshaw SM, Bellomo R (2012) Clinical review: volume of fluid resuscitation and the incidence of acute kidney injury - a systematic review. Crit Care 16:230

11. Malbrain MLNG, Marik PE, Witters I et al (2014) Fluid overload, de-resuscitation, and outcomes in critically ill or injured patients: a systematic review with suggestions for clinical practice. Anaesthesiol Intensive Ther 46:361-380

12. Byrne L, Obonyo NG, Diab SD et al (2018) Unintended consequences: fluid resuscitation worsens shock in an ovine model of endotoxemia. Am J Respir Crit Care Med 198:1043-1054

13. García MIM, González PG, Romero MG et al (2015) Effects of fluid administration on arterial load in septic shock patients. Intensive Care Med 41:1247-1255

14. Ince C (2015) Hemodynamic coherence and the rationale for monitoring the microcirculation. Crit Care 19:58

15. Monnet X, Marik P, Teboul J-L (2016) Passive leg raising for predicting fluid responsiveness: a systematic review and meta-analysis. Intensive Care Med 42:1935-1947

16. Miller A, Mandeville J (2016) Predicting and measuring fluid responsiveness with echocardiography. Echo Res Pract 3:G1-G12

17. Jacobs R, Lochy S, Malbrain MLNG (2019) Phenylephrine-induced recruitable preload from the venous side. J Clin Monit Comput 33:373-376

18. Hjortrup PB, Haase N, Bundgaard $\mathrm{H}$ et al (2016) Restricting volumes of resuscitation fluid in adults with septic shock after initial management: the CLASSIC randomised, parallel-group, multicentre feasibility trial. Intensive Care Med 42:1695-1705

19. Russell JA, Gordon AC, Walley KR (2019) Early may be better: early low-dose norepinephrine in septic shock. Am J Respir Crit Care Med 199:1049-1051

20. Van Regenmortel N, Verbrugghe W, Roelant E et al (2018) Maintenance fluid therapy and fluid creep impose more significant fluid, sodium, and chloride burdens than resuscitation fluids in critically ill patients: a retrospective study in a tertiary mixed ICU population. Intensive Care Med 44:409-417

21. Cordemans C, De laet I, Van Regenmortel N et al (2012) Aiming for a negative fluid balance in patients with acute lung injury and increased intra- abdominal pressure: a pilot study looking at the effects of PALtreatment. Ann Intensive Care 2:S15

22. Martin GS, Moss M, Wheeler AP et al (2005) A randomized, controlled trial of furosemide with or without albumin in hypoproteinemic patients with acute lung injury. Crit Care Med 33:1681-1687 